Preprint typeset in JHEP style. - HYPER VERSION

SHEP 06-17

\title{
Structure of the MHV-rules Lagrangian
}

\author{
James H. Ettle and Tim R. Morris \\ School of Physics and Astronomy, University of Southampton, Highfield, \\ Southampton SO17 1BJ, U.K. \\ E-mails: jhe@phys.soton.ac.ul, T.R.Morris@soton.ac.uk
}

\begin{abstract}
Recently, a canonical change of field variables was proposed that converts the Yang-Mills Lagrangian into an MHV-rules Lagrangian, i.e. one whose tree level Feynman diagram expansion generates CSW rules. We solve the relations defining the canonical transformation, to all orders of expansion in the new fields, yielding simple explicit holomorphic expressions for the expansion coefficients. We use these to confirm explicitly that the three, four and five point vertices are proportional to MHV amplitudes with the correct coefficient, as expected. We point out several consequences of this framework, and initiate a study of its implications for MHV rules at the quantum level. In particular, we investigate the wavefunction matching factors implied by the Equivalence Theorem at one loop, and show that they may be taken to vanish in dimensional regularisation.
\end{abstract}




\section{Contents}

1. Introduction 1

2. Notation and Transformation 4

$2.1 \quad$ Preliminaries 1

2.2 The transformation 5

2.3 The precise correspondence 7

3. Explicit Expansion 9

3.1 A expansion coefficients to all orders 9

$3.2 \bar{A}$ expansion coefficients to all orders

3.3 Three-point vertex 13

3.4 Four-point vertices 13

3.5 Five-point vertices 14

4. Equivalence Theorem Matching Factors 15

5. Conclusions 18

A. Off-shell matching factors 20

\section{Introduction}

The standard approach to computing perturbative scattering amplitudes is to develop a Feynman diagram expansion using Feynman rules, which in turn follow directly from a bare Lagrangian. While this procedure is very well understood [1], the complexity of the calculation grows so rapidly with increasing order that it seriously challenges our ability, especially at one loop and higher, to compute background processes to the accuracy that must be known if we are to exploit fully the new physics potential of the LHC [2].

Stemming from two remarkable papers [3, 4], dramatically simpler methods have been developed which could help solve this problem. These so far apply mostly to scattering amplitudes in gauge theories at tree level $[5,6]$ but also to some one-loop amplitudes [7-10].

The starting point is the set of maximally helicity-violating (MHV) amplitudes: the tree-level colour-ordered partial amplitudes for $n_{-}=2$ negative helicity gluons 
and any number $n_{+} \geq 1$ of positive helicity gluons. ${ }^{1}$ Despite the factorial growth in complexity of the underlying Feynman diagrams for increasing $n_{+}$, when these are written in terms of some associated two-component spinors, the amplitudes collapse to a single simple ratio [11]. As if this were not astonishing enough, considerations of topological string theory in twistor space [3] led Cachazo, Svrcek and Witten to conjecture that arbitrary $\left(n_{+}, n_{-}\right)$tree-level amplitudes of gluons may be calculated by sowing together certain off-shell continuations of these MHV amplitudes with scalar propagators, using colour-ordered Feynman rules [4]. These "MHV-rules" (a.k.a. CSW rules) result in much simpler expressions for generic small $n_{-}$, growing in complexity only polynomially with increasing $n_{+}$. Under parity, we can exchange $n_{+} \leftrightarrow n_{-}$, resulting in an alternative expansion via $\overline{\mathrm{MHV}}$-rules.

The MHV rules were proven indirectly as a consequence of another development [12]: the BCFW recursion, an expansion of colour-ordered amplitudes involving simultaneously both MHV and $\overline{\mathrm{MHV}}$ sub-amplitudes, which in some cases leads to even more compact expressions albeit at the expense of introducing unphysical poles in intermediate terms. The recursion equation results from using Cauchy's theorem on a carefully chosen complex continuation, to reconstruct the amplitude from its poles. This idea has been generalised to provide a direct proof of the MHV rules [13], and applied and extended at tree-level to both Yang-Mills and other theories [6]. It has also been generalised to one-loop amplitudes $[7,9]$, although the appearance of physical cuts, spurious cuts, higher poles in complex momenta, and the need for regularisation in general, limits the power of this idea.

In a separate and initially unexpected development, the MHV rules have been applied successfully at one loop $[9,10,14]$ by again tying together the same off-shell continuation of the MHV amplitudes with scalar propagators. This is meant to provide the cut-constructible parts of one-loop amplitudes (which is the whole oneloop amplitude in theories with unbroken supersymmetry). Although much evidence supports this contention, a full proof has been missing [15] — until now (see below).

The cut-constructible parts of one-loop amplitudes are specified because these are directly related to tree amplitudes via their cuts. No claim is made therefore to generate the full one-loop amplitude in non-supersymmetric theories from MHV rules. Indeed, it is known that certain non-constructible (parts of) one-loop amplitudes are not generated by MHV rules [16], a fact that we will return to in the conclusions.

As can be gleaned even from this short survey, a feature of these new developments is that they lie outside the Lagrangian framework, proceeding by a combination of inspired conjecture and varying levels of proof.

All this potentially changes with Mansfield's paper however [17]. According to this paper, a change of field variables satisfying certain specific properties trans-

\footnotetext{
${ }^{1}$ Throughout the paper we label all external momenta as out-going.
} 
forms the standard Lagrangian into an equivalent MHV-rules Lagrangian, i.e. one involving effectively a complex scalar whose vertices are proportional to the CSW off-shell continuation of the MHV amplitudes, so that it directly generates at tree level the CSW rules. (For a different approach to such a Lagrangian, see ref. [18]. For approaches via (ambi)twistor space see ref. [19].)

Assuming the validity of this proposal, we can apply at once the well developed quantum field theory framework [1] to confirm and extend these methods. For example, if we side-step for the moment the issue of regularisation, by concentrating on the cut-constructible parts of one-loop amplitudes at non-exceptional momenta, the existence of the MHV-rules Lagrangian shows immediately that the program of refs. $[9,10,14,15]$ is correct because the application of MHV rules at one loop simply means in this framework that one constructs the one loop amplitudes by using this equivalent Lagrangian. (Actually, we need to take into account 'ET matching factors', certain wave function renormalization matching factors that arise from applying the equivalence theorem $[1,20]$, however we will see later that they vanish.)

It also means that, up to modifications which are necessary to provide a full regularisation (which is also much easier to figure out within standard quantum field theory) and the ET matching factors, the MHV rules must work for the full amplitudes at any number of loops. In particular we can expect that MHV rules supply the full amplitude at any loop in finite supersymmetric theories $($ e.g. $\mathcal{N}=4$ Yang-Mills).

We make further comments about this framework in the conclusions.

The structure of the rest of the paper is as follows. In the next section we briefly review Mansfield's construction [17], which is based on light-cone quantization, and state precisely the form the MHV-rules Lagrangian should take, paying attention to conventions. We note in passing that the arbitrary null vector introduced by Cachazo, Svrcek and Witten to define off-shell continuations is exactly the one defining lightcone time.

In sec. 3, we give the transformation explicitly to all orders by deriving recurrence relations, which we then solve. We find that the expansion coefficients take simple forms and are in particular already holomorphic. From Mansfield's arguments it follows that the resulting vertices yield the MHV-rules Lagrangian, however we nevertheless check explicitly that the required three, four and five point vertices are obtained.

In sec. 4, we investigate the one-loop ET matching factors. These are examples of terms that could not be anticipated using the earlier methods. In the massless case however (as here) it is easy to argue that they vanish in dimensional regularisation. Although we are missing the full regularisation, the leading divergent pieces should be able to be trusted. We check explicitly and find indeed that they are forced to vanish.

Finally, in section sec. 5, we draw our conclusions. 


\section{Notation and Transformation}

In this section we review the form of the transformation to an MHV-rules Lagrangian as proposed in ref. [17]. We define closely allied notation, but pin down several factors involved in comparison with the MHV rules.

\subsection{Preliminaries}

Mansfield maps from the Minkowski coordinates $\left(t, x^{1}, x^{2}, x^{3}\right)$ using a $(+,-,-,-)$ signature metric to ones appropriate for light-front quantization i.e. quantization surfaces of constant $x^{0}=\mu \cdot x$, where $\mu^{\nu}$ is some constant null-vector. Defining Minkowski coordinates so that $\mu^{\nu}=(1,0,0,1)$, the map is

$$
x^{0}=t-x^{3}, \quad x^{\overline{0}}=t+x^{3}, \quad z=x^{1}+i x^{2}, \quad \bar{z}=x^{1}-i x^{2} .
$$

In these coordinates, the metric has covariant components $g_{0 \overline{0}}=g_{\overline{0} 0}=-g_{z \bar{z}}=$ $-g_{\bar{z} z}=1 / 2$, all others being zero.

We will mostly deal with covariant vectors (1-forms) for which it is useful to introduce a more compact notation, thus we write $\left(p_{0}, p_{\overline{0}}, p_{z}, p_{\bar{z}}\right) \equiv(\check{p}, \hat{p}, p, \bar{p})$. This allows us, in sympathy with the literature, to write components of external momenta simply by the number of the leg with the appropriate decoration, thus the $n^{\text {th }}$ momentum $p_{n}^{\mu}$ will simply be written as $(\check{n}, \hat{n}, \tilde{n}, \bar{n})$. Note that we put a tilde over the $z$ component in this case, so that $\tilde{n}$ will not be confused with a numerical factor of $n$ [see e.g. (2.4,2.5)].

We can write any 4 -vector in the form of a bispinor with components $p_{\alpha \dot{\alpha}}$ as

$$
\left(p_{\alpha \dot{\alpha}}\right)=\left(p^{t} \delta_{\alpha \dot{\alpha}}+\mathbf{p} \cdot \boldsymbol{\sigma}_{\alpha \dot{\alpha}}\right)=2\left(\begin{array}{cc}
\check{p} & -p \\
-\bar{p} & \hat{p}
\end{array}\right),
$$

where $\boldsymbol{\sigma}$ is the usual 3 -vector formed of the Pauli matrices. If $p^{\mu}$ is null, $\check{p} \hat{p}=p \bar{p}$ and the bispinor factorises: $p_{\alpha \dot{\alpha}}=\lambda_{\alpha} \tilde{\lambda}_{\dot{\alpha}}$. For real $\left(p^{t}, \mathbf{p}\right), \lambda_{\alpha}$ and $\tilde{\lambda}_{\dot{\alpha}}$ are related by complex conjugation. For momenta, it is helpful to make the choice:

$$
\lambda=\sqrt{2}\left(\begin{array}{c}
-p / \sqrt{\hat{p}} \\
\sqrt{\hat{p}}
\end{array}\right) \quad \text { and } \quad \tilde{\lambda}=\sqrt{2}\left(\begin{array}{c}
-\bar{p} / \sqrt{\hat{p}} \\
\sqrt{\hat{p}}
\end{array}\right)
$$

Of particular importance for MHV rules is the 'angle bracket' invariant, which we can now express as

$$
\langle 12\rangle:=\epsilon^{\alpha \beta} \lambda_{1 \alpha} \lambda_{2 \beta}=2 \frac{(12)}{\sqrt{\hat{1} \hat{2}}},
$$

where the two-dimensional alternating tensor has $\epsilon^{12}=1$, and we have introduced

$$
(12) \equiv\left(\mathbf{p}_{1} \mathbf{p}_{2}\right):=\hat{1} \tilde{2}-\hat{2} \tilde{1}
$$


We can similarly express the contragredient invariant $\left[\lambda_{1} \lambda_{2}\right]:=\epsilon^{\dot{\alpha} \dot{\beta}} \tilde{\lambda}_{1 \dot{\alpha}} \tilde{\lambda}_{2 \dot{\beta}}$ in terms of the complex conjugate $2\{12\} / \sqrt{\hat{1} \hat{2}}$, where

$$
\{12\}:=(12)^{*}=\hat{1} \overline{2}-\hat{2} \overline{1} .
$$

Choice (2.3) is not suitable for $\mu_{\alpha \dot{\alpha}}=\nu_{\alpha} \tilde{\nu}_{\dot{\alpha}}$, since the only non-zero covariant component is $\check{\mu}=1$. Thus from (2.2) we take instead

$$
\nu=\tilde{\nu}=\left(\begin{array}{c}
\sqrt{2} \\
0
\end{array}\right) .
$$

The standard polarisations for a massless on-shell vector boson of momentum $p$,

$$
E_{+}=\frac{\nu \tilde{\lambda}}{\langle\nu \lambda\rangle} \quad \text { and } \quad E_{-}=\frac{\lambda \tilde{\nu}}{[\lambda \nu]}
$$

then have non-zero components

$$
E_{+}=-\frac{1}{2}, \quad \bar{E}_{-}=\frac{1}{2} .
$$

There are also $\check{E}_{+}=-\bar{p} / 2 \hat{p}$ and $\check{E}_{-}=p / 2 \hat{p}$, although these time-like components will not be needed. (The remaining components vanish.)

Importantly, (2.3) contains no reference to $\check{p}$ and makes sense even for non-null momenta. In this case, the spinors factor the bispinor associated to the null momentum $p+a \mu$, where $a=p \bar{p} / \hat{p}-\check{p}$. This definition is equivalent to the CSW prescription for taking the spinors off-shell $[4,17]$, providing the CSW spinor is identified (projectively) with $\nu$. Indeed, the CSW prescription is to introduce a fixed spinor $\eta$ and take the off-shell momentum spinor to be proportional to

$$
\epsilon^{\dot{\alpha} \dot{\beta}} p_{\alpha \dot{\alpha}} \tilde{\eta}_{\dot{\beta}}=\lambda_{\alpha}[\lambda \eta]-a \nu_{\alpha}[\nu \eta],
$$

so the two definitions coincide when $\eta \propto \nu$. We thus arrive at the satisfying conclusion that the arbitrary null vector $\eta_{\alpha} \tilde{\eta}_{\dot{\alpha}}$ is just $\mu_{\alpha \dot{\alpha}}$, the vector defining light-cone time and the quantization surface.

\subsection{The transformation}

We take the Yang-Mills action written as [17]

$$
S=\frac{1}{2 g^{2}} \int d t d x^{1} d x^{2} d x^{3} \operatorname{tr} F^{\lambda \rho} F_{\lambda \rho},
$$

where

$$
F_{\lambda \rho}=\left[D_{\lambda}, D_{\rho}\right], \quad D_{\mu}=\partial_{\mu}+A_{\mu}, \quad A_{\mu}=A_{\mu}^{a} T^{a},
$$


and the generators of the internal group have been taken as anti-Hermitian:

$$
\left[T^{a}, T^{b}\right]=f^{a b c} T^{c}, \quad \operatorname{tr}\left(T^{a} T^{b}\right)=-\frac{1}{2} \delta^{a b}
$$

We choose light-cone gauge $\hat{A}=0$, discarding the non-interacting Fadeev-Popov ghosts, and integrate out the longitudinal field $\check{A}$ (which appears quadratically and is not dynamical, in the sense that the Lagrangian has no terms $\check{\partial} \check{A}$ ).

The resulting action takes the form

$$
S=\frac{4}{g^{2}} \int d x^{0} L
$$

where $L$ is the light-cone Lagrangian defined as an integral $d^{3} \mathbf{x}=d x^{\overline{0}} d z d \bar{z}$ over surfaces $\Sigma$ of constant $x^{0}$. From (2.7), $A(\bar{A})$ has only positive (negative) helicity on-shell states. Labelling the parts by the participating helicities $L=L^{-+}+L^{++-}+$ $L^{--+}+L^{\prime--++}$, where

$$
\begin{aligned}
L^{-+}[A] & =\operatorname{tr} \int_{\Sigma} d^{3} \mathbf{x} \bar{A}(\check{\partial} \hat{\partial}-\partial \bar{\partial}) A \\
L^{++-}[A] & =-\operatorname{tr} \int_{\Sigma} d^{3} \mathbf{x}\left(\bar{\partial} \hat{\partial}^{-1} A\right)[A, \hat{\partial} \bar{A}] \\
L^{--+}[A] & =-\operatorname{tr} \int_{\Sigma} d^{3} \mathbf{x}[\bar{A}, \hat{\partial} A]\left(\partial \hat{\partial}^{-1} \bar{A}\right) \\
L^{\prime--++}[A] & =-\operatorname{tr} \int_{\Sigma} d^{3} \mathbf{x}[\bar{A}, \hat{\partial} A] \hat{\partial}^{-2}[A, \hat{\partial} \bar{A}] .
\end{aligned}
$$

Now we define a canonical change of variables from $A$ to $B$ to absorb the unwanted term $L^{++-}$into the kinetic term:

$$
L^{-+}[A]+L^{++-}[A]=L^{-+}[B]
$$

This means that the transformation is performed on the quantization surface $\Sigma$ with all fields having the same time dependence $x^{0}$ (which henceforth we suppress). It induces the following transformation for the canonical momenta:

$$
\hat{\partial} \bar{A}^{a}(\mathbf{y})=\int_{\Sigma} d^{3} \mathbf{x} \frac{\delta B^{b}(\mathbf{x})}{\delta A^{a}(\mathbf{y})} \hat{\partial} \bar{B}^{b}(\mathbf{x})
$$

Substituting this back into (2.16) yields the defining relation between $A$ and $B$ :

$$
\int_{\Sigma} d^{3} \mathbf{y}\left[D, \frac{\bar{\partial}}{\hat{\partial}} A\right]^{a}(\mathbf{y}) \frac{\delta B^{b}(\mathbf{x})}{\delta A^{a}(\mathbf{y})}=\frac{\partial \bar{\partial}}{\hat{\partial}} B^{b}(\mathbf{x}) .
$$

It follows that $A$ is a power series in $B$, of the form $A=B+O\left(B^{2}\right)$ (at least for general momenta: see further comments in sec. 3) and thus from (2.17), $\bar{A}$ is a power series in $B$ each term containing also a single $\bar{B}$, of the form $\bar{A}=\bar{B}+O(\bar{B} B)$. 
Thus it follows from the equivalence theorem that we can equally well use $B(\bar{B})$ as the positive (negative) helicity field in place of $A(\bar{A})[1,17]$. The canonical nature of the transformations ensures that the change of variables in the functional integral has unit jacobian [17], whilst substituting the transformations into $L$ results, from (2.16) and (2.14,2.15), in a Lagrangian with an infinite number of interactions each containing just two $\bar{B}$ fields and an increasing number of $B$ fields:

$$
L=L^{-+}[B]+L^{--+}[B]+L^{--++}[B]+L^{--+++}[B]+\cdots .
$$

This has precisely the structure required to be identified with the MHV-rules Lagrangian, in the sense that its tree level perturbation theory generates CSW rules with the Feynman rules following from these vertices. It follows immediately that these vertices when taken on-shell must be proportional to the corresponding MHV amplitude since only one vertex is used to construct the tree-level amplitude with the right helicity assignment of two negative and any number positive helicities. (Using two or more vertices results in " $\mathrm{N}^{n} \mathrm{MHV}$ " amplitudes with more than two negative helicities.)

It remains to show that when off-shell, these vertices must give the CSW continuations of the MHV amplitudes. Mansfield argues that this would follow from the fact that the vertices contain no explicit $x^{0}$ dependence (or $\breve{\partial}$ ) if one can show that the vertices are also holomorphic in the sense that they contain no $\bar{\partial}$ derivatives. We will see that this is true at least for general momenta. Mansfield argues that this holomorphy follows from considering the homogeneous transformations:

$$
\delta A=[A, \theta] \quad \text { and } \quad \delta \bar{A}=[\bar{A}, \theta],
$$

where $\theta$ is a function of $\bar{z}$ only. He uses the fact that this corresponds to the shift $\delta \bar{\partial} A=[A, \bar{\partial} \theta]$ when acting on (2.16), the equation defining the expansion, and does not leave it invariant, whereas $(2.20)$ does leave invariant the sum of the two terms (2.14,2.15) which generates all the vertices in (2.19). From this viewpoint however, it is a surprise to find that the coefficients of the series for $A(\bar{A})$ themselves are already holomorphic. Furthermore, we will show that they take a very simple form.

\subsection{The precise correspondence}

Clearly, it would be very welcome to investigate explicitly the transformation (2.17,2.18) and its effect on the vertices. In order to do this, we write the general $n$-point term in (2.19) in 3-momentum space as

$$
\frac{1}{2} \sum_{s=2}^{n} \int_{12 \cdots n} V_{12 \cdots n}^{s} \operatorname{tr}\left[\bar{B}_{\overline{1}} B_{\overline{2}} \cdots \bar{B}_{\bar{s}} \cdots B_{\bar{n}}\right]
$$

where the bar on the indices indicates that the 3 -momentum dependence is $B_{\bar{k}} \equiv$ $B\left(-\mathbf{p}_{k}\right)$ (we continue to suppress their common $x^{0}$ dependence, which is not Fourier 
transformed), the missing fields in the trace being $B_{\bar{k}} \mathrm{~s}$. The components of $\mathbf{p}$ are expressed, as always, as $(\hat{p}, p, \bar{p})$. The integral shorthand means, here and later,

$$
\int_{12 \cdots n} \equiv \prod_{k=1}^{n} \int \frac{d \hat{p}_{k} d p_{k} d \bar{p}_{k}}{(2 \pi)^{3}},
$$

and the vertex is expressed as

$$
V_{12 \cdots n}^{s}=(2 \pi)^{3} \delta^{3}\left(\mathbf{p}_{1}+\mathbf{p}_{2}+\cdots+\mathbf{p}_{n}\right) V^{s}\left(\mathbf{p}_{1}, \mathbf{p}_{2}, \cdots, \mathbf{p}_{n}\right) .
$$

Throughout the paper, expansion coefficients, vertices and amplitudes carry these momentum conserving delta-functions; they will be factored off in this manner and thus not written explicitly. We often simply write $V^{s}(12 \cdots n) \equiv V^{s}\left(\mathbf{p}_{1}, \mathbf{p}_{2}, \cdots, \mathbf{p}_{n}\right)$. It should be borne in mind that from (2.22) such coefficients are only defined when their momentum arguments sum to zero.

Note that, by using (2.10), we can always express the group theory factors as traces of products of the $B \mathrm{~s}$ and $\bar{B}$ s valued in the Lie algebra, as in (2.21). This form leads to the required colour-ordered Feynman rules. We will express group theory factors throughout by absorbing them in the fields, including for the expansion coefficients of sec. 3 .

By the cyclicity of the trace, we can always arrange for the first field in (2.21) to be a $\bar{B}$. Since we have a choice of two $\bar{B}$ s, we could have restricted the sum in (2.21) to $r \leq\lfloor n / 2+1\rfloor$, however by writing it as a full sum over $r$ and dividing the result by two, we get the same thing except that the $\lfloor n / 2+1\rfloor^{\text {th }}$ vertex is accompanied by a factor $\frac{1}{2}$ when $n$ is even, consistent with the fact that it alone has a $Z_{2}$ symmetry under exchange of the $\bar{B}$ s.

We would like to compare $V_{1 \cdots n}^{s}$ to the MHV amplitude [11]:

$$
A_{n}=g^{n-2} \frac{\langle r s\rangle^{4}}{\langle 12\rangle\langle 23\rangle \cdots\langle n-1, n\rangle\langle n 1\rangle},
$$

$r$ and $s$ being the negative helicity legs, itself a component of the full tree-level amplitude:

$$
\sum_{\sigma} \operatorname{tr}\left(T^{a_{\sigma(1)}} \cdots T^{a_{\sigma(n)}}\right)(2 \pi)^{4} i \delta^{4}\left(p^{1}+\cdots+p^{n}\right) A_{n}^{\sigma},
$$

the sum being over distinct cyclic orderings $\sigma$. However this is written with different conventions.

There are many ways to perform the translation. Perhaps the following is simplest. The normalization for the generators in (2.23, 2.24) is such that $\operatorname{tr}\left(T^{a} T^{b}\right)=\delta^{a b}$ and $\left[T^{a}, T^{b}\right]=i \sqrt{2} f^{a b c} T^{c}[21]$. Comparing with (2.10) we see we need to replace $T^{a} \mapsto-i T^{a} / \sqrt{2}$. To form the momentum space Feynman rule from (2.21) and (2.22), we Fourier transform the $x^{0}$ dependence also, obtaining a four-dimensional delta-function as in (2.24), however in our case it is defined via (2.1) to be

$$
(2 \pi)^{4} \delta^{4}(p)=\int d x^{0} d x^{\overline{0}} d z d \bar{z} \mathrm{e}^{i\left(\check{p} x^{0}+\hat{p} x^{\overline{0}}+p z+\bar{p} \bar{z}\right) .}
$$


As can be seen by computing the jacobian or $\sqrt{-g}$, this is four times the one in (2.24). Noting that $p^{\mu} p_{\mu}=4(\check{p} \hat{p}-p \bar{p})$, we see that (2.11) and (2.12) yield the propagator $2 i g^{2} \delta^{a b} / p^{2}$. To bring this to canonical normalization requires absorbing $2 g^{2}$ by $B \mapsto B g \sqrt{2}$ (similarly $\bar{B}$ ). Combining all these with the prefactor from (2.11), we see that the $r=1 \mathrm{MHV}$ amplitude should be given by

$$
\frac{16}{g^{2}}(-i g)^{n} V^{s}(12 \cdots n)\left(E^{+}\right)^{n-2}\left(\bar{E}^{-}\right)^{2} .
$$

Of course the factor $\frac{1}{2}$ for the even- $n\lfloor n / 2+1\rfloor^{\text {th }}$ vertex in (2.21) is cancelled here by the two ways to form this amplitude. Finally, from (2.7), (2.23) and (2.4) we thus expect to find

$$
\begin{aligned}
V^{s}(12 \cdots n) & =(2 i)^{n-4} \frac{\langle 1 s\rangle^{4}}{\langle 12\rangle\langle 23\rangle \cdots\langle n-1, n\rangle\langle n 1\rangle} \\
& =i^{n} \frac{\hat{2} \cdots \hat{n}(1 s)^{4}}{\hat{1} \hat{s}^{2}(12)(23) \cdots(n-1, n)(n 1)}
\end{aligned}
$$

\section{Explicit Expansion}

In this section we derive recursion relations for the expansions satisfying (2.18) and (2.17) in the case that $A$ and $B$ have support only on general momenta. We then solve these and use the results to confirm (2.25) for the three point, the two four-point and the two five-point vertices.

\subsection{A expansion coefficients to all orders}

Rearranging (2.18) and transforming to 3-momentum space yields

$$
\omega_{1} A_{1}-i \int_{23} \zeta_{3}\left[A_{2}, A_{3}\right](2 \pi)^{3} \delta^{3}\left(\mathbf{p}_{1}-\mathbf{p}_{2}-\mathbf{p}_{3}\right)=\int_{\mathbf{p}} \omega_{\mathbf{p}} B_{\mathbf{p}}^{b} \frac{\delta A_{1}}{\delta B_{\mathbf{p}}^{b}},
$$

using the notation set up in subsec. 2.3, and introducing $\zeta_{\mathbf{p}} \equiv \zeta(\mathbf{p})=\bar{p} / \hat{p}$ and $\omega_{\mathbf{p}}=p \bar{p} / \hat{p}$.

From this one would be tempted to conclude that at lowest order $A_{\mathbf{p}}=B_{\mathbf{p}}$ and thus in general, absorbing the group theory generators into the fields, $A$ has an expansion in $B$ of the form

$$
A_{1}=\sum_{n=2}^{\infty} \int_{2 \cdots n} \Upsilon_{12 \cdots n} B_{\overline{2}} \cdots B_{\bar{n}}
$$

where $\Upsilon(\mathbf{p},-\mathbf{p})=1$. However we have to divide through by $\omega_{\mathbf{p}}$ to conclude that $A_{\mathbf{p}}=$ $B_{\mathbf{p}}$ to lowest order, and since $\omega_{\mathbf{p}}$ vanishes when $p=0$ or $\bar{p}=0$, more general solutions exist where $A_{\mathbf{p}}$ has a piece not containing $B_{\mathbf{p}}$ but with delta-function support, viz. $\delta(\bar{p})$ and/or $\delta(p)$. 
It is tempting to ignore these terms, however we cannot do so and also implement some expected residual gauge invariances: the light cone action (2.11) is arrived at by fixing the gauge $\mu \cdot A \propto \hat{A}=0$. This leaves gauge transformations $\delta A_{\mu}=\left[D_{\mu}, \theta\right]$ unfixed providing $\theta$ does not depend on $\hat{z}$. If $\theta$ depends on $x^{0}$, we can expect the form of the gauge transformation to be modified as a result of $\check{A}$ being integrated out. On the other hand, it is easy to check that (2.11) is invariant under holomorphic gauge transformations $\theta(z)$, providing we interpret ${ }^{2} \bar{\partial} \hat{\partial}^{-1} \theta:=\hat{\partial}^{-1}(\bar{\partial} \theta)=0$. Similarly (by symmetry) we also have antiholomorphic gauge invariance generated by $\theta(\bar{z})$.

Now, the left hand side of (2.16) is actually invariant under holomorphic gauge transformations which indicates that $B$ and $\bar{B}$ must also be invariant. Indeed this ensures that the defining transformations (2.17,2.18) transform covariantly. However, (3.2) only transforms covariantly if we allow for an extra term independent of $B$ and proportional to $\delta\left(\bar{p}_{1}\right)$ to absorb the gauge transformation. This indicates one should really interpret (3.2) as amounting to further gauge fixing. For the present we will simply declare that (3.2) is to be applied only for generic momenta in particular such that $p_{1}$ and $\bar{p}_{1}$ are non-zero.

Substituting (3.2) in (3.1), comparing coefficients and stripping off momentum conserving delta functions as in (2.22), yields the recurrence relation:

$$
\Upsilon(1 \cdots n)=\frac{i}{\omega_{1}+\cdots+\omega_{n}} \sum_{j=2}^{n-1}\left(\zeta_{j+1, n}-\zeta_{2, j}\right) \Upsilon(-, 2, \cdots, j) \Upsilon(-, j+1, \cdots, n),
$$

where the arguments labelled "-" are minus the sum of the remaining arguments (as follows from momentum conservation) and we have defined $\zeta_{j, k}=\zeta\left(P_{j, k}\right)$ with $P_{j, k}=\sum_{i=j}^{k} \mathbf{p}_{i}$ (similarly for $\omega_{j, k}$ below). The next two coefficients are thus

$$
\begin{aligned}
\Upsilon(123) & =i \frac{\zeta_{3}-\zeta_{2}}{\omega_{1}+\omega_{2}+\omega_{3}}, \\
\Upsilon(1234) & =\frac{1}{\omega_{1}+\omega_{2}+\omega_{3}+\omega_{4}}\left[\frac{\left(\zeta_{4}-\zeta_{3}\right)\left(\zeta_{3,4}-\zeta_{2}\right)}{\omega_{3,4}-\omega_{3}-\omega_{4}}+\frac{\left(\zeta_{4}-\zeta_{2,3}\right)\left(\zeta_{3}-\zeta_{2}\right)}{\omega_{2,3}-\omega_{2}-\omega_{3}}\right] .
\end{aligned}
$$

Although apparently not holomorphic, once they are expressed in terms of independent momenta and simplified, all $\bar{p}_{k}$ dependence drops out, resulting in very compact expressions:

$$
\begin{aligned}
\Upsilon(123) & =-i \frac{\hat{1}}{(23)}, \\
\Upsilon(1234) & =\frac{\hat{1} \hat{3}}{(23)(34)}, \\
\Upsilon(12345) & =i \frac{\hat{1} \hat{3} \hat{4}}{(23)(34)(45)} .
\end{aligned}
$$

\footnotetext{
${ }^{2}$ We note that this is consistent with the Mandelstam-Leibbrandt prescription [22].
} 
We have displayed the four-point coefficient which we also checked explicitly. Now we prove by induction that the general coefficient takes the form

$$
\Upsilon(1 \cdots n)=i^{n} \frac{\hat{1} \hat{3} \hat{4} \cdots \widehat{n-1}}{(23)(34) \cdots(n-1, n)},
$$

where $n \geq 4$. It is sufficient to show that substituting (3.6) into the right hand side of (3.3) yields the left hand side. Substituting (3.6) into the right hand side yields

$$
-\frac{\Upsilon(1 \cdots n)}{\hat{1}\left(\omega_{1}+\cdots+\omega_{n}\right)} \sum_{j=2}^{n-1} \frac{(j, j+1)}{\hat{j} \widehat{j+1}}\left\{P_{2, j} P_{j+1, n}\right\},
$$

after due care with the ends of the sum. Expanding the $(j, j+1)$ term and relabelling so that both halves are indexed by $j$ converts the sum to

$$
\sum_{j=2}^{n} \frac{\tilde{j}}{\hat{j}}\left(\left\{P_{2, j-1} P_{j, n}\right\}-\left\{P_{2, j} P_{j+1, n}\right\}\right),
$$

where contributions at the end of the sum are correctly incorporated by defining $P_{j, k}=0$ when $j>k$. Writing $P_{2, j}=P_{2, j-1}+\mathbf{p}_{j}$ and $P_{j+1, n}=-P_{2, j-1}-\mathbf{p}_{1}-\mathbf{p}_{j}$, and using the antisymmetry of (2.6), the sum collapses to $-\hat{1}\left(\omega_{1}+\cdots+\omega_{n}\right)$, proving the assertion (3.6).

\section{2 $\bar{A}$ expansion coefficients to all orders}

Differentiating (3.2) with respect to $B$ and substituting the inverse into (2.17) yields an expansion for $\bar{A}$ of the form

$$
\hat{1} \bar{A}_{\overline{1}}=\sum_{m=2}^{\infty} \sum_{s=2}^{m} \int_{2 \cdots m} \hat{s} \Xi_{\overline{1} 2 \cdots m}^{s-1} B_{\overline{2}} \cdots \bar{B}_{\bar{s}} \cdots B_{\bar{m}},
$$

where the superscript on $\Xi$ labels the relative position (not momentum) of the $\bar{B}$ field and the missing fields are $B_{\bar{k}} s$. We use the invariant

$$
\operatorname{tr} \int_{\Sigma} d^{3} \mathbf{x} \check{\partial} A \hat{\partial} \bar{A}=\operatorname{tr} \int_{\Sigma} d^{3} \mathbf{x} \check{\partial} B \hat{\partial} \bar{B}
$$

to extract a recurrence relation. Recalling that all fields have the same $x^{0}$ dependence, we have from (3.2),

$$
\check{\partial} A_{1}=\sum_{n=2}^{\infty} \sum_{r=2}^{n} \int_{2 \cdots n} \Upsilon_{12 \cdots n} B_{\overline{2}} \cdots \check{\partial} B_{\bar{r}} \cdots B_{\bar{n}} .
$$

Substituting this and (3.7) into the above, using cyclicity of the trace and several careful relabellings we find:

$$
\begin{array}{r}
\Xi^{l}(1 \cdots n)=-\sum_{r=2}^{n+1-l} \sum_{m=\max (r, 3)}^{r+l-1} \Upsilon(-, n-r+3, \cdots, m-r+1) \times \\
\Xi^{l+r-m}(-, m-r+2, \cdots, n-r+2),
\end{array}
$$


where $l=1, \cdots, n-1$, the momentum indices on the right hand side must be interpreted cyclically, i.e. $\bmod n$, and $\Xi^{1}(\mathbf{p},-\mathbf{p})=1$. [Note that $m$ is the number of arguments in $\Upsilon$. The inner sum should be interpreted as zero when $r=2$ and $l=1$, alternatively the lower limit in $r$ can be given as $\max (2,4-l)$.] From this, or directly, one can readily compute the first few coefficients:

$$
\begin{aligned}
\Xi^{1}(123) & =-\Upsilon(231), \\
\Xi^{2}(123) & =-\Upsilon(312), \\
\Xi^{1}(1234) & =-\Upsilon(2+3,4,1) \Xi^{1}(1+4,2,3)-\Upsilon(2341), \\
\Xi^{2}(1234) & =-\Upsilon(3+4,1,2) \Xi^{1}(1+2,3,4)-\Upsilon(2+3,4,1) \Xi^{2}(1+4,2,3)-\Upsilon(3412), \\
\Xi^{3}(1234) & =-\Upsilon(3+4,1,2) \Xi^{2}(1+2,3,4)-\Upsilon(4123) .
\end{aligned}
$$

Clearly, since the $\Upsilon$ coefficients are already holomorphic, the $\Xi$ s will turn out to be also. In fact they take a very simple form when expressed in terms of $\Upsilon$ :

$$
\Xi^{s-1}(1 \cdots n)=-\frac{\hat{s}}{\hat{1}} \Upsilon(1 \cdots n)
$$

$(s=2, \cdots, n$ and $n \geq 2)$.

Let us now prove this assertion. Again, it is sufficient to show that substituting (3.9) into the right hand side of (3.8) yields its left hand side. Substituting (3.9) into the right hand side and using (3.6), we find we can extract a factor of $\Xi^{l}(1 \cdots n)$ and thus learn that proving $(3.9)$ is equivalent to proving that

$$
\sum_{r=2}^{n+1-l} \sum_{m=\max (r, 3)}^{r+l-1} \frac{(m-r+1, m-r+2)(n-r+2, n-r+3) \hat{P}_{n-r+3, m-r+1}}{\widehat{m-r+1} \widehat{m-r+2} \widehat{n-r+2} \widehat{n-r+3}}
$$

equals

$$
-\frac{(12)(n 1)}{\hat{1} \hat{2} \hat{n}}
$$

In a similar way to the proof of (3.6), we now expand the factor $(m-r+1, m-r+2)$ and relabel so that both halves are collected. (However since here momentum labels are treated $\bmod n, \hat{P}_{j, k}$ means summing from $j$ increasing to $k$, going through 1 when $k<j$.) This allows us to perform the inner sum with the result displayed in braces:

$$
\sum_{r=2}^{n+1-l} \frac{(n-r+2, n-r+3)}{\widetilde{n-r+2} 2 \widetilde{n-r+3}}\left\{-\tilde{P}_{q+2, l}+\widetilde{\frac{l+1}{\widehat{l+1}}} \hat{P}_{n-r+3, l}-\widetilde{\widetilde{q+1}} \hat{P}_{n-r+3, q+1}\right\} \text {. }
$$

Here $q=1$ if $r=2$ otherwise $q=0$. We note that the term in curly brackets vanishes when $r=2$ and $l=1$, as required, $c f$. below (3.9). At this stage it proves useful to consider the case $l=n-1$ separately. Substituting this into (3.11) gives

$$
\frac{(n 1)}{\hat{1} \hat{n}}\left\{\tilde{n}+\tilde{1}+\tilde{2}-\tilde{n}-\frac{\tilde{2}}{\hat{2}} \hat{P}_{1,2}\right\},
$$


where we have used momentum conservation on the first two terms in curly brackets. It is immediate to see that this gives (3.10) as required. For $l<n-1$, we expand $(n-r+2, n-r+3)$ and collect both halves. This gives

$$
\begin{aligned}
& \tilde{P}_{1, l+1}\left(\frac{\tilde{1}}{\hat{1}}-\widetilde{\frac{l+1}{\widehat{l+1}}}\right)+\frac{\tilde{n}}{\hat{n}}\left(\frac{\hat{1} \tilde{2}}{\hat{2}}-\tilde{1}\right)+\frac{\tilde{1}}{\hat{1}}\left(-\tilde{P}_{3, l}+\widetilde{\widetilde{l+1}} \hat{P}_{1, l}-\tilde{2}-\frac{\hat{1} \tilde{2}}{\hat{l}}\right) \\
& +\widetilde{\frac{l+1}{\widetilde{l+1}}}\left(\tilde{P}_{2, l}+\widetilde{l+1}+\frac{\tilde{1}}{\hat{1}} \hat{P}_{l+2,1}\right) \text {. }
\end{aligned}
$$

Using momentum conservation and cancelling terms, this expression collapses to (3.10), and thus (3.9) is proved.

Recall that to obtain the vertices $(2.21,2.22)$ in $(2.19)$, we substitute the series (3.2) and (3.7), which we have seen are holomorphic, into (2.14) and (2.15), which are also holomorphic. Thus we have proven that the vertices in (2.19) are holomorphic. It follows by Mansfield's arguments [17] that these vertices when off-shell give the CSW continuations of the MHV amplitudes, i.e. are the ones in (2.25). Nevertheless it is instructive to verify that this really does work out in practice.

\subsection{Three-point vertex}

Since $A(\bar{A})$ is, to lowest order, linear in $B(\bar{B})$, the three-point vertex is simply the light-cone gauge vertex (2.14). Transforming to 3 -momentum space and casting in the form (2.21), we have

$$
V^{2}(123)=i \frac{\hat{3}}{\hat{1} \hat{2}}(21) .
$$

On the other hand, from (2.25) we expect

$$
-i \frac{\hat{3}}{\hat{1} \hat{2}} \frac{(12)^{3}}{(23)(31)} .
$$

Substituting $\mathbf{p}_{3}=-\mathbf{p}_{1}-\mathbf{p}_{2}$ in the denominator, we readily see that these equations are the same and thus simply verify that the light-cone gauge three-point vertex satisfies the general formula (2.25) as expected:

$$
V^{2}(123)=\frac{1}{2 i} \frac{\langle 12\rangle^{3}}{\langle 23\rangle\langle 31\rangle}
$$

\subsection{Four-point vertices}

The four-point vertices receive contributions from (2.14) and the first non-trivial terms in (3.2,3.7), and also directly from (2.15), and thus are more exacting tests. We write (2.15) in the same form as (2.21):

$$
L^{\prime--++}=\int_{1234}\left\{W_{1234}^{2} \operatorname{tr}\left[\bar{A}_{\overline{1}} \bar{A}_{\overline{2}} A_{\overline{3}} A_{\overline{4}}\right]+\frac{1}{2} W_{1234}^{3} \operatorname{tr}\left[\bar{A}_{\overline{1}} A_{\overline{2}} \bar{A}_{\overline{3}} A_{\overline{4}}\right]\right\}
$$


where

$$
W^{2}(1234)=-\frac{\hat{1} \hat{3}+\hat{2} \hat{4}}{(\hat{1}+\hat{4})^{2}} \quad \text { and } \quad W^{3}(1234)=\frac{\hat{1} \hat{4}+\hat{2} \hat{3}}{(\hat{1}+\hat{2})^{2}}+\frac{\hat{1} \hat{2}+\hat{3} \hat{4}}{(\hat{1}+\hat{4})^{2}}
$$

(after symmetrization). Therefore,

$$
V^{2}(1234)=\frac{\hat{1}}{\hat{5}} V^{2}(523) \Xi^{2}(\overline{5} 41)+\frac{\hat{2}}{\hat{5}} V^{2}(154) \Xi^{1}(\overline{5} 23)+V^{2}(125) \Upsilon(\overline{5} 34)+W^{2}(1234),
$$

where the momentum $\mathbf{p}_{5}$ is determined by conservation in each term (thus e.g. in the first term $\mathbf{p}_{5}=\mathbf{p}_{1}+\mathbf{p}_{4}$ ). To compare this formula to the expected result (2.25) we map both to unique functions of independent momenta. For example, we substitute for the last momentum: $\mathbf{p}_{4}=-\mathbf{p}_{1}-\mathbf{p}_{2}-\mathbf{p}_{3}$. It is then straightforward using computer algebra to show that this coincides with the right hand side of (2.25), and thus:

$$
V^{2}(1234)=\frac{\langle 12\rangle^{3}}{\langle 23\rangle\langle 34\rangle\langle 41\rangle}
$$

For example, simplifying by partial fractions, both (2.25) and (3.13) give:

$$
\frac{(\hat{1}+\hat{2})^{2}(\hat{1} \tilde{2}-\hat{2} \tilde{1})}{\hat{1} \hat{2}[(\hat{1}+\hat{2}) \tilde{3}-\hat{3} \tilde{1}-\hat{3} \tilde{2}]}-\frac{\hat{2}(\hat{1}+\hat{2}+\hat{3})(\hat{1} \tilde{2}-\hat{2} \tilde{1})}{\hat{1}(\hat{2}+\hat{3})(\hat{2} \tilde{3}-\hat{3} \tilde{2})}-\frac{\hat{1} \hat{3}(\hat{1} \tilde{2}-\hat{2} \tilde{1})}{\hat{2}(\hat{2}+\hat{3})(\hat{1} \tilde{2}+\hat{1} \tilde{3}-\hat{2} \tilde{1}-\hat{3} \tilde{1})}
$$

Similarly, after symmetrization,

$$
\begin{aligned}
V^{3}(1234)= & \frac{\hat{1}}{\hat{5}} V^{2}(352) \Xi^{2}(\overline{5} 41)+\frac{\hat{3}}{\hat{5}} V^{2}(512) \Xi^{1}(\overline{5} 34) \\
& +\frac{\hat{3}}{\hat{5}} V^{2}(154) \Xi^{2}(\overline{5} 23)+\frac{\hat{1}}{\hat{5}} V^{2}(534) \Xi^{1}(\overline{5} 12)+W^{3}(1234),
\end{aligned}
$$

and it is straightforward to confirm as above that this agrees with (2.25):

$$
V^{3}(1234)=\frac{\hat{2} \hat{4}}{\hat{1} \hat{3}} \frac{(13)^{4}}{(12)(23)(34)(41)}
$$

\subsection{Five-point vertices}

The five-point vertices leave no doubt that the off-shell MHV vertices are produced: they involve substituting up to the first three terms in the expansions (3.2, 3.7) into 
both original vertices $(2.14,2.15)$. We find

$$
\begin{aligned}
V^{2}(12345) & =\frac{\hat{3}}{\hat{6}} V^{2}(612) \Xi^{1}(\overline{6} 345)+\frac{\hat{1}}{\hat{\gamma}} V^{2}(726) \Upsilon(\overline{6} 34) \Xi^{2}(\overline{7} 51)+V^{2}(126) \Upsilon(\overline{6} 345) \\
& +\frac{\hat{2}}{\hat{6}} \frac{\hat{1}}{\hat{\gamma}} V^{2}(764) \Xi^{1}(\overline{6} 23) \Xi^{2}(\overline{7} 51)+\frac{\hat{2}}{\hat{6}} V^{2}(167) \Xi^{1}(\overline{6} 23) \Upsilon(\overline{7} 45) \\
& +\frac{\hat{2}}{\hat{6}} V^{2}(165) \Xi^{1}(\overline{6} 234)+W^{2}(1236) \Upsilon(\overline{6} 45)+W^{2}(1265) \Upsilon(\overline{6} 34) \\
& +\frac{\hat{2}}{\hat{6}} W^{2}(1645) \Xi^{1}(\overline{6} 23)+\frac{\hat{1}}{\hat{6}} W^{2}(6234) \Xi^{2}(\overline{6} 51), \\
V^{3}(12345)= & \frac{\hat{3}}{\hat{6}} V^{2}(612) \Xi^{1}(\overline{6} 345)+\frac{\hat{1}}{\hat{6}} V^{2}(634) \Xi^{2}(\overline{6} 512)+\frac{\hat{1}}{\hat{6}} V^{2}(637) \Xi^{1}(\overline{6} 12) \Upsilon(\overline{7} 45) \\
& +\frac{\hat{1}}{\hat{6}} \frac{\hat{3}}{\hat{\gamma}} V^{2}(675) \Xi^{1}(\overline{6} 12) \Xi^{1}(\overline{7} 34)+\frac{1}{\hat{3}} \frac{\hat{6}}{\hat{6}} V^{2}(672) \Xi^{1}(\overline{6} 34) \Xi^{2}(\overline{7} 51) \\
& +\frac{\hat{1} \hat{3}}{\hat{6}} \frac{\hat{7}}{2} V^{2}(674) \Xi^{2}(\overline{6} 51) \Xi^{2}(\overline{7} 23)+\frac{\hat{3}}{\hat{6}} V^{2}(167) \Xi^{2}(\overline{6} 23) \Upsilon(\overline{7} 45) \\
& +\frac{\hat{3}}{\hat{6}} V^{2}(165) \Xi^{2}(\overline{6} 234)+\frac{\hat{1}}{\hat{6}} V^{2}(362) \Xi^{3}(\overline{6} 451)+\frac{\hat{3}}{\hat{6}} W^{2}(1645) \Xi^{2}(\overline{6} 23) \\
& +\frac{\hat{1}}{\hat{6}} W^{2}(6345) \Xi^{1}(\overline{6} 12)+\frac{\hat{3}}{\hat{6}} W^{3}(1265) \Xi^{2}(\overline{6} 34)+\frac{\hat{1}}{\hat{6}} W^{3}(3462) \Xi^{2}(\overline{6} 51) \\
& +W^{3}(1236) \Upsilon(\overline{6} 45)
\end{aligned}
$$

(where, like before, indices 6 and 7 label momenta that are uniquely determined in terms of the first five by momentum conservation). Again, eliminating $\mathbf{p}_{5}$ in favour of the first four momenta and doing likewise for the corresponding right hand sides in (2.25), we find the expressions agree, and thus confirm that

$$
\begin{aligned}
& V^{2}(12345)=2 i \frac{\langle 12\rangle^{3}}{\langle 23\rangle\langle 34\rangle\langle 45\rangle\langle 51\rangle}, \quad \text { and } \\
& V^{3}(12345)=2 i \frac{\langle 13\rangle^{4}}{\langle 12\rangle\langle 23\rangle\langle 34\rangle\langle 45\rangle\langle 51\rangle} .
\end{aligned}
$$

\section{Equivalence Theorem Matching Factors}

Even if a change of field variables from $A$ to $B$ turns the light-cone gauge Lagrangian into an MHV-rules Lagrangian, this does not mean necessarily that one-loop and higher contributions are obtained purely by using the CSW rules. We have to remember that wavefunction renormalization terms, which we have already referred to as ET matching factors, are also generated [1,20].

Let us recall that an S-matrix element is obtained by computing the amputated Green function (the LSZ procedure). Thus to the action (2.11), we should add the 
source terms

$$
\int d^{4} x J^{a} \bar{A}^{a}+\bar{J}^{a} A^{a}
$$

where, after amputating propagators $\langle A \bar{A}\rangle(p)$, by multiplying by $-i p^{2}$ and taking the on-shell limit $p^{2} \rightarrow 0$, we see that $J$ acts as a source for positive helicity $A$ legs in the amputated Green function (and likewise $\bar{J}$ for negative helicity $\bar{A}$ legs).

Now, substituting the series (3.2) and (3.7) into (4.1), we see that at tree-level the process of multiplying by $-i p^{2}$ and taking the on-shell limit, kills all terms but the first, which survives because these generate cancelling poles via the propagators $\langle B \bar{B}\rangle(p)$. Therefore, we can effectively write the source terms as

$$
\int d^{4} x J^{a} \bar{B}^{a}+\bar{J}^{a} B^{a} .
$$

This is the tree-level content of the Equivalence Theorem.

At one loop and higher, the only change to this conclusion is that we can use the vertices in the expansions (3.2,3.7) and those of the Lagrangian, viz. (2.21), to form self-energy-like diagrams, $c f$. fig. 1. Since these again attach to propagators carrying the external momentum $p^{\mu}$, they survive the process of amputation.

These ET matching factors are not explicitly Lorentz invariant, a consequence of both the light-cone axial gauge and the change to $B$ fields. However, if everything is defined correctly, Lorentz invariance should be recovered in the process of forming S-matrix elements. Therefore we should find that these matching factors depend only on $p^{2}$, which is sent to zero. Since these self-energy-like diagrams then depend on no scale at all, in dimensional regularisation we must set them to zero.

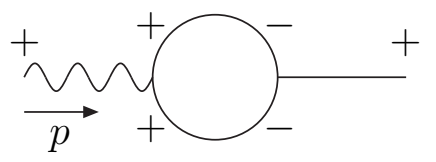

(a)

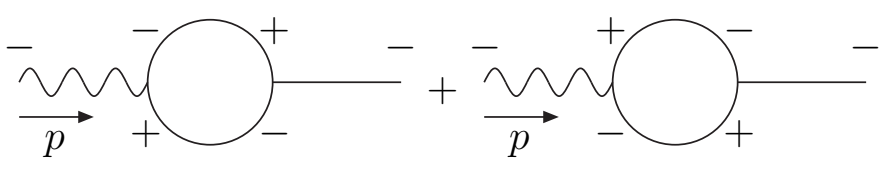

(b)

Figure 1: Topology of the one-loop ET matching factors, with (a) the contribution to negative helicity $\bar{B}$ legs; and (b) the contribution to positive helicity $B$ legs. Wavy lines denote $A$ and $\bar{A}$ fields, straight lines $B$ and $\bar{B}$.

We now confirm these conclusions at one loop. The terms in fig. 1 contribute by multiplying the legs of the corresponding tree-level amplitude (computed using the CSW rules). The diagrams in fig. 1 contain both ultraviolet and infrared divergences. We derived the vertices (3.2,3.7) and (2.21) without using any regularisation. Strictly speaking, we should therefore wait until a corresponding Lagrangian is supplied incorporating sufficient regularisation. However, if we proceed with the calculation, 
naïvely applying dimensional regularisation where needed, we can expect to get right the most divergent pieces.

From the diagram fig. 1(a), using (3.2) and (3.12), we obtain

$$
-\frac{1}{2} g^{2} C_{A} \int \frac{d^{4} q}{(2 \pi)^{4}} \frac{\Upsilon(-p, p+q,-q) V^{2}(q,-p-q, p)}{q^{2}(p+q)^{2}}=-i \hat{p} g^{2} C_{A} \int \frac{d^{4} q}{(2 \pi)^{4}} \frac{1}{q^{2}(p+q)^{2} \hat{q}}
$$

where a factor $4 g^{2}$ arises from the multiplier in (2.11), a factor $1 / 4$ arises for converting $d q d \bar{q} d \hat{q} d \check{q}$ to the $d q^{t} d q^{1} d q^{2} d q^{3}$ taken above, and $C_{A} / 2$ arises from evaluating the trace over the various products of generators, $C_{A}$ being the adjoint Casimir [so $C_{A}=N$ for $\left.S U(N)\right]$. The right hand side follows after using partial fractions and shifts in loop momentum. We can write it in Lorentz invariant fashion using $\mu$, and furthermore map it to $D=4-2 \epsilon$ dimensions:

$$
-i g^{2} C_{A} \mu \cdot p \int \frac{d^{D} q}{(2 \pi)^{D}} \frac{1}{q^{2}(p+q)^{2} \mu \cdot q} .
$$

In order to evaluate the integral we need to keep $p$ off-shell and let $p^{2} \rightarrow 0$ at the end. This is consistent with the way this contribution arises through the LSZ procedure.

The integral has already been evaluated in ref. [23] and we reproduce the result in the appendix. However we can see that it vanishes in the $p^{2} \rightarrow 0$ limit without calculation. By Lorentz invariance, the result can only depend on $p^{2}$ and $\mu \cdot p\left(\mu^{2}\right.$ being zero). However since the expression is independent of $r$ when scaled as $\mu \mapsto r \mu$, we see that the result does not in fact depend on $\mu \cdot p$. We thus confirm that the integral depends only on the scale $p^{2} \rightarrow 0$ and thus must be set to zero, as required in dimensional regularisation. ${ }^{3}$

Now we turn to the two diagrams of fig. 1(b). Using (3.7) and (3.12), we obtain

$$
\begin{aligned}
& \frac{1}{2} g^{2} C_{A} \int \frac{d^{4} q}{(2 \pi)^{4}} \frac{1}{q^{2}(p+q)^{2}}\left(1+\frac{\hat{q}}{\hat{p}}\right) \Upsilon(p+q,-q,-p) V^{2}(p, q,-p-q) \\
- & \frac{1}{2} g^{2} C_{A} \int \frac{d^{4} q}{(2 \pi)^{4}} \frac{1}{q^{2}(p+q)^{2}} \frac{\hat{q}}{\hat{p}} \Upsilon(-q,-p, p+q) V^{2}(-p-q, p, q),
\end{aligned}
$$

which simplifies to

$$
-i g^{2} C_{A} \int \frac{d^{4} q}{(2 \pi)^{4}} \frac{1}{q^{2}(p+q)^{2}} \frac{(\hat{p}+\hat{q})^{3}}{\hat{q} \hat{p}^{2}} .
$$

Writing this in a Lorentz invariant way in $D$ dimensions gives

$$
-i g^{2} C_{A} \int \frac{d^{D} q}{(2 \pi)^{D}} \frac{1}{q^{2}(p+q)^{2}} \frac{(\mu \cdot p+\mu \cdot q)^{3}}{\mu \cdot q(\mu \cdot p)^{2}} .
$$

\footnotetext{
${ }^{3}$ In more detail, by dimensions the integral depends only on $p^{2}$ through the factor $\left(-p^{2}\right)^{-\epsilon}$. The vanishing of the integral is then fully justified if we keep $\epsilon<0$ as $p \rightarrow 0$.
} 
This integral is also evaluated off shell in the appendix, however again we see that this expression is independent of $\mu \cdot p$ and thus depends only on the scale $p^{2}$, forcing the integral to vanish on shell, in dimensional regularisation.

Finally we would like to note that in this case the contribution contains the term

$$
\frac{11}{6} \frac{g^{2} C_{A}}{(4 \pi)^{2}} \frac{1}{\epsilon}\left(-p^{2}\right)^{-\epsilon},
$$

arising from an ultraviolet divergence. It is tempting to try and interpret this in the case where $p^{2}$ is small but non-zero, since if we add the divergence to the $B$ tree-level matching factor (which is just $Z_{+}=1$ ), it is precisely of the correct form to be cancelled by renormalizing a factor of the bare coupling constant $g_{0}$, such as appears

in the tree-level MHV-rules amplitude. The left-over $\ln \left(-p^{2}\right)$ would be expected to cancel such a term in tree-level bremsstrahlung from off-shell positive helicity gluons. However this cannot be the whole story. In particular we expect other divergences in the one-loop amplitudes obtained using MHV-rules.

\section{Conclusions}

We have seen that the direct series solution of the transformation proposed in ref. [17] does indeed yield the vertices (2.25) necessary to reproduce the CSW rules at tree level.

A key step in making the expansions manageable was to recognize that all the group theory factors could be absorbed into the fields, allowing also the derivation of compact recurrence relations (3.3, 3.8). We were able to solve these and thus discovered very simple expressions (3.6, 3.9) for the coefficients of the expansion of $A$ and $\bar{A}$, in terms of the "MHV-frame" $B$ field to all orders. We expect that these explicit expressions will be useful for further developments in the subject. Of particular note is that the expansion coefficients are purely holomorphic - a property we would like to understand on a more profound basis than we do at present. It follows that all the Lagrangian vertices (2.21) are also holomorphic. We can then use the arguments as given in ref. [17] to prove that all these vertices must correspond to the CSW off-shell extension of MHV-rules vertices.

We would like to mention that the comparison between the explicitly derived vertices that come out of the expansion and MHV vertices was very straightforward to do algebraically once the spinors were converted into momentum components using (2.3) and (2.4). This is because it is then straightforward to eliminate one of the momentum arguments by using momentum conservation and from there obtain a unique representation of the vertex function. It is surely the case that such a procedure would allow verification of algebraic (rather than just numerical) equivalence for different representations of other amplitudes in the literature. 
In the light of what we have learned, it is obvious that lying behind the CSW rules is light-cone quantization. The fixed spinor $\eta$ introduced in ref. [4] defines a preferred null direction which we have seen is the same direction as the null direction $\mu$ defining light-cone time in light-cone quantization. We note that it is the locality of the resulting vertices in light-cone time which is actually exploited when applying the Feynman Tree Theorem in ref. [15].

It is difficult to overstress the potential importance of the program started by ref. [17]. The spinor/twistor methods have been very successful at tree level and for cut-constructible pieces at one loop. This is the natural domain for methods inherently tied to the structure of tree-level four-dimensional on-shell scattering processes. Progress has been limited beyond this domain (see however ref. [25]).

However, since it transpires that these methods are a direct consequence of a change of field variables, extending these methods simply means applying well understood techniques from quantum field theory. For example, it is now obvious that the CSW rules apply to fully off-shell amplitudes, the external spinors being continued off-shell using the CSW prescription.

It also can no longer be in doubt that these methods can be appropriately regularised (for computing general quantum corrections): we can simply apply our favourite method to the Yang-Mills Lagrangian and trace through the consequences for the change of variables. The real question is whether a regularisation can be applied (hopefully dimensional regularisation appropriately adapted) in such a way as to preserve sufficiently the simplifying power of these methods.

These regularised CSW methods will then apply to the whole amplitude to any number of loops, excepting only that we must multiply by the correct ET matching factors. Of course, as is well known, we cannot compute the $n$-point one-loop all "+" amplitude or the $n$-point amplitude with just one negative helicity leg, using only MHV rules [16]. These amplitudes are non-vanishing but finite [26] and of course contain no cut-constructible part. It is thus a reasonable assumption that these amplitudes are missing simply because the extra regularisation structure is missing.

Another possible extension is to develop mixed representations for computing amplitudes, where gluonic parts can be computed in the "MHV frame" by performing the relevant change of variables, while particles' interactions that do not benefit from this (e.g. involving massive particles) can be computed in the normal "Feynman rules frame". It is even possible to consider mixed representations for amplitudes containing the same field.

In sec. 1 , we investigated the quantum contributions to the ET matching factors, which are $Z_{+}=Z_{-}=1$ at tree level, and which multiply S-matrix elements computed using CSW rules. These are two-point contributions formed from the expansion coefficients (3.6,3.9) and the MHV-rules vertices (2.25). Although Lorentz invariance has been broken by the light-cone gauge and the change of field variables, it should be recovered when computing S-matrix elements. Therefore the matching factors 
can only depend on the external momentum squared of the scattered particle, so providing we are dealing with only massless particles, the one-loop and higher-loop contributions depend on no scale at all and must be set to zero in dimensional regularisation. We verified this argument for the divergent parts at one loop, giving also explicit off-shell expressions for these factors. We note that in general certain on-shell contributions to the ET matching factors could prove to be non-zero when massive particles are included in the theory.

\section{Acknowledgments}

It is a pleasure to thank Andi Brandhuber, Nick Evans, Nigel Glover, Valya Khoze, Paul Mansfield, Simon McNamara, Douglas Ross, Olly Rosten, Bill Spence and Gabriele Travaglini for discussions, and PPARC for financial support. Special thanks

to Andi, Bill and Gabriele for bringing to our attention ref. [23], correcting an error in an earlier version of this paper.

\section{A. Off-shell matching factors}

We confirm the result [23]

$$
\frac{1}{\epsilon^{2}} g^{2} C_{A} c_{\Gamma}\left(-p^{2}\right)^{-\epsilon}
$$

for integral (4.3), where the standard one-loop factor $c_{\Gamma}$ is

$$
c_{\Gamma}=\frac{\Gamma(1+\epsilon) \Gamma^{2}(1-\epsilon)}{(4 \pi)^{2-\epsilon} \Gamma(1-2 \epsilon)} .
$$

Temporarily ignoring $-i g^{2} C_{A}$ and combining denominators in (4.3) using Feynman parameters we have

$$
4 \mu \cdot p \int \frac{d^{D} q}{(2 \pi)^{D}} d \alpha d \beta d \gamma \delta(1-\alpha-\beta-\gamma) \frac{1}{\left[\alpha q^{2}+\beta(p+q)^{2}+2 \gamma \mu \cdot q\right]^{3}} .
$$

We note in passing that this has only infrared divergences. Performing the momentum integral, the $\alpha$ integral, and substituting $\beta=\rho(1-\omega)$ and $\gamma=\rho \omega$, yields

$$
-2 i \mu \cdot p \frac{\Gamma(1+\epsilon)}{(4 \pi)^{2-\epsilon}} \int_{0}^{1} d \rho \int_{0}^{1} d \omega \rho^{-\epsilon}(1-\omega)^{-1-\epsilon}(1-\rho \omega)^{-1+2 \epsilon}\left\{2 \rho \omega \mu \cdot p-(1-\rho) p^{2}\right\}^{-1-\epsilon} .
$$

As noted below (4.3), this integral does not in fact depend on $\mu \cdot p$. Exploiting this [23], we set $\mu \cdot p>0$, substitute $\omega \mapsto \omega / \mu \cdot p$ and then let $\mu \cdot p \rightarrow \infty$. The result,

$$
-2 i \frac{\Gamma(1+\epsilon)}{(4 \pi)^{2-\epsilon}} \int_{0}^{1} d \rho \int_{0}^{\infty} d \omega \rho^{-\epsilon}\left\{2 \rho \omega-(1-\rho) p^{2}\right\}^{-1-\epsilon}
$$

is readily evaluated and gives (A.1) on reinstating $-i g^{2} C_{A}$. 
Expanding the numerator in (4.5), the $(\mu \cdot p)^{3}$ term is identical to (4.3) and thus gives (A.1). The other terms do not have $\mu \cdot q$ on the denominator and are therefore straightforward to evaluate. (For $p$ off-shell, they have only ultraviolet divergences.) The result for (4.5) is therefore

$$
\frac{1}{\epsilon^{2}} g^{2} C_{A} c_{\Gamma}\left(-p^{2}\right)^{-\epsilon}+\frac{1}{\epsilon} g^{2} C_{A} c_{\Gamma} \frac{11-7 \epsilon}{(6-4 \epsilon)(1-2 \epsilon)}\left(-p^{2}\right)^{-\epsilon} .
$$

\section{References}

[1] C. Itzykson and J. B. Zuber, "Quantum Field Theory" McGraw-Hill, 1980.

[2] E. W. N. Glover, Nucl. Phys. Proc. Suppl. 116 (2003) 3 [arXiv:hep-ph/0211412]; C. Anastasiou, L. J. Dixon, K. Melnikov and F. Petriello, Phys. Rev. D 69 (2004) 094008 [arXiv:hep-ph/0312266].

[3] E. Witten, Commun. Math. Phys. 252, 189 (2004) [arXiv:hep-th/0312171].

[4] F. Cachazo, P. Svrcek and E. Witten, JHEP 0409, 006 (2004) [arXiv:hep-th/0403047].

[5] G. Georgiou and V. V. Khoze, JHEP 0405, 070 (2004) [arXiv:hep-th/0404072]; G. Georgiou, E. W. N. Glover and V. V. Khoze, JHEP 0407, 048 (2004) [arXiv:hepth/0407027]; J. B. Wu and C. J. Zhu, JHEP 0407, 032 (2004) [arXiv:hep-th/0406085]; J. B. Wu and C. J. Zhu, JHEP 0409, 063 (2004) [arXiv:hep-th/0406146]; X. Su and J. B. Wu, Mod. Phys. Lett. A 20, 1065 (2005) [arXiv:hep-th/0409228]. I. Bena, Z. Bern and D. A. Kosower, Phys. Rev. D 71, 045008 (2005) [arXiv:hep-th/0406133]; D. A. Kosower, Phys. Rev. D 71, 045007 (2005) [arXiv:hep-th/0406175]; S. Giombi, R. Ricci, D. Robles-Llana and D. Trancanelli, JHEP 0407, 059 (2004) [arXiv:hepth/0405086]; L. J. Dixon, E. W. N. Glover and V. V. Khoze, JHEP 0412, 015 (2004) [arXiv:hep-th/0411092]; D. Belov and G. W. Moore, arXiv:hep-th/0412167. S. D. Badger, E. W. N. Glover and V. V. Khoze, JHEP 0503, 023 (2005) [arXiv:hepth/0412275]; T. G. Birthwright, E. W. N. Glover, V. V. Khoze and P. Marquard, JHEP 0505, 013 (2005) [arXiv:hep-ph/0503063]; T. G. Birthwright, E. W. N. Glover, V. V. Khoze and P. Marquard, JHEP 0507, 068 (2005) [arXiv:hep-ph/0505219]; P. Marquard and T. G. Birthwright, arXiv:hep-ph/0505264; X. d. Ji, J. P. Ma and F. Yuan, JHEP 0507, 020 (2005) [arXiv:hep-ph/0503015]; R. Britto, F. Cachazo and B. Feng, Nucl. Phys. B 715, 499 (2005) [arXiv:hep-th/0412308].

[6] M. x. Luo and C. k. Wen, JHEP 0503, 004 (2005) [arXiv:hep-th/0501121]; M. x. Luo and C. k. Wen, Phys. Rev. D 71, 091501 (2005) [arXiv:hep-th/0502009]; J. Bedford, A. Brandhuber, B. J. Spence and G. Travaglini, Nucl. Phys. B 721, 98 (2005) [arXiv:hep-th/0502146]; F. Cachazo and P. Svrcek, [arXiv:hep-th/0502160]; R. Britto, B. Feng, R. Roiban, M. Spradlin and A. Volovich, Phys. Rev. D 71, 105017 (2005) [arXiv:hep-th/0503198]; S. D. Badger, E. W. N. Glover, V. V. Khoze and P. Svrcek, JHEP 0507, 025 (2005) [arXiv:hep-th/0504159]; S. D. Badger, E. W. N. Glover and V. V. Khoze, JHEP 0601, 066 (2006) [arXiv:hep-th/0507161]; D. Forde and D. A. Kosower, Phys. Rev. D 73 (2006) 065007 [arXiv:hep-th/0507292]. 
[7] Z. Bern, L. J. Dixon and D. A. Kosower, Phys. Rev. D 71, 105013 (2005) [arXiv:hepth/0501240; M. Bertolini, F. Bigazzi and A. L. Cotrone, Phys. Rev. D 72, 061902 (2005) [arXiv:hep-th/0505055]; M. Bertolini, F. Bigazzi and A. L. Cotrone, Phys. Rev. D 72, 061902 (2005) [arXiv:hep-th/0505055]; Z. Bern, N. E. J. Bjerrum-Bohr, D. C. Dunbar and H. Ita, JHEP 0511 (2005) 027 [arXiv:hep-ph/0507019].

[8] R. Britto, F. Cachazo and B. Feng, Nucl. Phys. B 725, 275 (2005) [arXiv:hepth/0412103]; S. J. Bidder, N. E. J. Bjerrum-Bohr, D. C. Dunbar and W. B. Perkins, Phys. Lett. B 612, 75 (2005) [arXiv:hep-th/0502028]; A. Brandhuber, S. McNamara, B. J. Spence and G. Travaglini, JHEP 0510, 011 (2005) [arXiv:hep-th/0506068]; E. I. Buchbinder and F. Cachazo, JHEP 0511, 036 (2005) [arXiv:hep-th/0506126]; K. Risager, S. J. Bidder and W. B. Perkins, JHEP 0510, 003 (2005) [arXiv:hepth/0507170]; Z. Bern, V. Del Duca, L. J. Dixon and D. A. Kosower, Phys. Rev. D 71, 045006 (2005) [arXiv:hep-th/0410224]; R. Roiban, M. Spradlin and A. Volovich, Phys. Rev. Lett. 94, 102002 (2005) [arXiv:hep-th/0412265].

[9] Z. Bern, L. J. Dixon and D. A. Kosower, Phys. Rev. D 72, 045014 (2005) [arXiv:hepth/0412210].

[10] A. Brandhuber, B. J. Spence and G. Travaglini, Nucl. Phys. B 706, 150 (2005) [arXiv:hep-th/0407214]; C. Quigley and M. Rozali, JHEP 0501, 053 (2005) [arXiv:hep-th/0410278]; J. Bedford, A. Brandhuber, B. J. Spence and G. Travaglini, Nucl. Phys. B 706, 100 (2005) [arXiv:hep-th/0410280]; J. Bedford, A. Brandhuber, B. J. Spence and G. Travaglini, Nucl. Phys. B 712, 59 (2005) [arXiv:hep-th/0412108];

[11] S. J. Parke and T. R. Taylor, Phys. Rev. Lett. 56, 2459 (1986); F. A. Berends and W. T. Giele, Nucl. Phys. B 306, 759 (1988).

[12] R. Britto, F. Cachazo, B. Feng and E. Witten, Phys. Rev. Lett. 94 (2005) 181602 [arXiv:hep-th/0501052].

[13] K. Risager, JHEP 0512 (2005) 003 [arXiv:hep-th/0508206].

[14] F. Cachazo, P. Svrcek and E. Witten, JHEP 0410, 077 (2004) [arXiv:hep-th/0409245].

[15] A. Brandhuber, B. Spence and G. Travaglini, JHEP 0601 (2006) 142 [arXiv:hepth/0510253].

[16] F. Cachazo, P. Svrcek and E. Witten, JHEP 0410 (2004) 074 [arXiv:hep-th/0406177].

[17] P. Mansfield, JHEP 0603, 037 (2006) [arXiv:hep-th/0511264].

[18] A. Gorsky and A. Rosly, JHEP 0601, 101 (2006) [arXiv:hep-th/0510111].

[19] L. J. Mason, JHEP 0510, 009 (2005) [arXiv:hep-th/0507269]; L. J. Mason and D. Skinner, Phys. Lett. B 636, 60 (2006) [arXiv:hep-th/0510262]; R. Boels, L. Mason and D. Skinner, arXiv:hep-th/0604040.

[20] M. C. Bergere and Y. M. Lam, Phys. Rev. D 13 (1976) 3247. 
[21] L. J. Dixon, lectures published in Boulder TASI 95:539-584 [arXiv:hep-ph/9601359].

[22] S. Mandelstam, Nucl. Phys. B 213 (1983) 149; G. Leibbrandt, Phys. Rev. D 29 (1984) 1699.

[23] D. A. Kosower and P. Uwer, Nucl. Phys. B 563 (1999) 477 [arXiv:hep-ph/9903515].

[24] Z. Bern, L. J. Dixon, D. C. Dunbar and D. A. Kosower, Nucl. Phys. B 425, 217 (1994) [arXiv:hep-ph/9403226].

[25] Z. Bern, L. J. Dixon and D. A. Kosower, Phys. Rev. D 71, 105013 (2005) [arXiv:hepth/0501240]; C. F. Berger, Z. Bern, L. J. Dixon, D. Forde and D. A. Kosower, arXiv:hep-ph/0604195.

[26] Z. Bern, G. Chalmers, L. J. Dixon and D. A. Kosower, Phys. Rev. Lett. 72, 2134 (1994) [arXiv:hep-ph/9312333]; Z. Bern, L. J. Dixon and D. A. Kosower, arXiv:hepth/9311026. 